\title{
CRECIMIENTO DE LA PRODUCTIVIDAD TOTAL DE LOS FACTORES EN COSTA RICA E INESTABILIDAD MACROECONÓMICA
}

\section{TOTAL FACTOR PRODUCTIVITY GROWTH IN COSTA RICA AND MACROECONO- MIC INSTABILITY.}

\author{
Edgar A. Robles ${ }^{1}$
}

Recibido: $13 / 08 / 2019$

Aprobado: 04/05/2021

\section{RESUMEN}

El presente trabajo utiliza la metodología de contabilidad de crecimiento, llamada TwoDeflator Approach, para estimar la productividad total de los factores (PTF) en Costa Rica entre 1960 y 2019. Los resultados indican que el 14 \% del crecimiento económico es explicado por el crecimiento de la PTF. El resto del crecimiento económico se asocia a las contribuciones del capital (52\%) y del trabajo (34\%), las cuales han sido positivas durante todas las décadas de este periodo. En cuanto a la contribución del trabajo, su aporte al crecimiento económico ha sido por la cantidad y no por la calidad de la mano de obra, con excepción del primer (1960-1969) y último periodo (2010-2019) en donde la calidad aportó positivamente al crecimiento económico. En términos de cambios, los resultados muestran que el $69 \%$ del cambio en la tasa de crecimiento durante el periodo está explicado por cambios en la PTF; el restante $31 \%$ se explica por cambios en las contribuciones de los factores de producción (trabajo y capital).

Una vez estimada la PTF, se utiliza un modelo GARCH para estimar la relación entre la instabilidad macroeconómica y el crecimiento de la PTF. Así, se encontró evidencia de que el crecimiento de la PTF en Costa Rica se ve afectado negativamente por inflaciones altas. Por su parte, el crecimiento de la PTF se beneficia de un mayor crecimiento de la apertura comercial, medida por la suma de importaciones y exportaciones a PIB, y se perjudica cuando existe una mayor volatilidad de la profundización financiera, medida por la razón de M1 o M2 al PIB.

PALABRAS CLAVE: PRODUCTIVIDAD TOTAL DE LOS FACTORES (PTF), CRECIMIENTO ECONÓMICO, INESTABILIDAD MACROECONÓMICA, ECONOMÍA DE COSTA RICA.

CLASIFICACIÓN JEL: 04, 05, E6.

\section{ABSTRACT}

This paper uses the growth accounting methodology, called Two- Deflator Approach, to estimate Total Factor Productivity (TFP) in Costa Rica between 1960 and 2019. The results 
Regarding the contribution of labor, its contribution to economic growth has been due to the quantity and not the quality of the workforce, except for the first (1960-1969) and last (20102019) periods, where quality contributed positively to economic growth. In terms of changes, the results show that $69 \%$ of the change in the growth rate during the period is explained by changes in TFP; the remaining $31 \%$ is explained by changes in the contributions of the factors of production (labor and capital).

Once the TFP is calculated, a GARCH model is used to estimate the relationship between the macroeconomic instability and TFP growth. Thus, evidence was found that TFP growth in Costa Rica is negatively affected by high inflation. Moreover, TFP growth benefits from higher openness to international trade, measured by the sum of imports and exports to GDP, and TFP is harmed when there is greater volatility of financial deepening, measured by the ratio from $\mathrm{M} 1$ or $\mathrm{M} 2$ to GDP.

KEYWORDS: TOTAL FACTOR PRODUCTIVITY, GROWTH ACCOUNTING, ECONOMIC GROWTH IN COSTA RICA

JEL CLASSIFICATION: 04, 05, E6.

\section{INTRODUCCIÓN}

El crecimiento económico medido por el incremento en el producto interno bruto (PIB) per cápita es un objetivo principal perseguido por todas las sociedades. Los datos muestran una asociación positiva entre el crecimiento del PIB y el crecimiento de la productividad total de los factores (PTF). Por definición, la PTF es el cambio en la producción que no está asociado a cambios en las cantidades o calidades de los insumos. Cuando el crecimiento de la PTF es alto, la tasa de crecimiento del PIB también lo es; mientras que las caídas en el crecimiento de la PTF se correlacionan con una disminución de las tasas de crecimiento del PIB. Esta relación ocurre porque la posibilidad de crecer con base en acumulación de insumos está limitada por la productividad marginal decreciente de ellos, por lo que el crecimiento permanente a largo plazo es posible solo cuando crece la PTF.

En consecuencia, es posible comprender el crecimiento del PIB encontrando los elementos que causan cambios positivos en la PTF. Por ello, un estudio integral del proceso de crecimiento económico debe incluir un análisis de las causas del crecimiento de la PTF.

Tinbergen (1942) inició una metodología para encontrar las causas del crecimiento económico, lo que hoy se conoce como las fuentes del crecimiento económico o contabilidad de crecimiento. Este procedimiento consiste en atribuir el crecimiento de la producción a incrementos en la cantidad de los insumos utilizados en el proceso productivo y a la PTF, medida como un residuo. El crecimiento económico, entonces, se explica por el análisis del comportamiento de sus componentes. Así, se considera que Tinbergen (1942) realizó la estimación del nivel de eficiencia de una economía, a pesar de que Stigler (1947) fue el primero que utilizó el concepto de PTF como una medida de eficiencia.

Posteriormente, Kendrick (1956, 1961), Kendrick y Grossman (1980) y Solow (1957) depuraron el método para estimar la PTF. Sin embargo, estos métodos incorporan solo los cambios en las cantidades de los insumos, por lo que la PTF puede interpretarse solo como un residuo de componentes de crecimiento inexplicables.

Harberger (1993) presenta una metodología más estilizada, denominada el Enfoque de dos deflactores (TDA). Con este método se permite una evaluación más completa de la contribución de la calidad del capital humano y así del factor trabajo al crecimiento. Este método tiene sus raíces en 
la teoría del capital y la PTF se interpreta como una reducción de costos reales, que es el elemento que explica el crecimiento económico.

Otro grupo amplio de literatura utiliza especificaciones paramétricas, semi paramétricas y no paramétricas para estimar la $\mathrm{PTF}^{2}$; sin embargo, estas metodologías poseen la desventaja de que requieren la especificación de una función de producción determinada que, por lo general, posee valores constantes para la proporción del producto que se asigna a la remuneración de cada uno de los factores. Esto tiende a provocar errores de medición en la PTF, principalmente cuando el estudio se realiza para un periodo amplio, en especial a causa de cambios significativos en la intensidad de uso de los factores asociados, por ejemplo, al proceso de apertura comercial que ha experimentado el mundo en los últimos 50 años.

Un estudio dentro de esta categoría fue realizado por Abarca y Ramírez (2016), utilizando una función para encontrar la evolución de la PTF de Costa Rica entre los años 1960 y 2014 . No obstante, como lo muestra Ivankovich y Martínez (2020), esta metodología tiende a arrojar menores valores de PTF para Costa Rica como se indicará más adelante en este artículo. Anterior a ellos, Rodríguez-Clare et. al (2003) utilizaron una especificación trans logarítmica para estimar la PTF de Costa Rica en el periodo 1961-2000.

El presente trabajo utiliza la metodología TDA para estimar la evolución de la productividad de la economía costarricense entre los años 1960 y 2019. En la sección 2, este artículo describe la metodología del TDA; en la 3 se presenta la información requerida para el cálculo. La sección 4 muestra los resultados obtenidos para el cálculo de la PTF en Costa Rica, durante el período de análisis. Por último, previo a las conclusiones, se presenta evidencia del efecto de la inestabilidad macroeconómica sobre el crecimiento de la PTF en Costa Rica, para lo cual se utiliza un modelo de inferencia estadística GARCH.

\section{METODOLOGÍA}

\section{El enfoque de dos deflactores para el cálculo de la PTF}

La metodología de Harberger $(1990,1993,1998)$ para identificar las fuentes del crecimiento económico se denomina el enfoque de dos deflactores (TDA) y proviene del uso de solo dos deflactores en el análisis. En lugar de utilizar muchos deflactores para expresar los productos $y$ los insumos en cantidades para cada sector económico, este enfoque utiliza un solo deflactor para expresar variables en términos de canastas reales de consumo o producción (PIB). Al mismo tiempo, en lugar de utilizar cientos de clases de trabajadores para incluir cambios en la calidad del capital humano en la contribución del factor trabajo al crecimiento, el TDA usa un segundo deflactor para expresar el trabajo en términos de unidades básicas. Entre las principales ventajas de esta metodología está no tener que disponer de una gran cantidad de información relacionada con la segmentación por tipos de trabajadores con, literalmente, cientos o miles de características para capturar las mejoras en la calidad del factor trabajo, lo cual tampoco aseguraría mayor exactitud en las estimaciones, pues es imposible definir de forma precisa todos los elementos asociados a cada trabajador. Además, en la mayoría de los países, esa segmentación de la información no existe, o no es comparable, sino hasta épocas recientes.

La metodología TDA se basa en la teoría del capital en oposición a la teoría de la producción. Bajo este enfoque, no hay necesidad de formulaciones explícitas de funciones de producción. Por el contrario, el análisis de la producción es visto como una evaluación a posterior de un proyecto social.

2 Por ejemplo, véase Ackerberg et al. (2015); Bauer (1990); Beveren (2010); Plastina y Lence (2018); y Tsionas y Polemis (2019); , entre otros. 
Todas las cantidades reales se expresan en términos de canastas agregadas utilizando el índice de precios al consumidor o el deflactor implícito del PIB. Si la inversión de cada año se expresa en términos del número de canastas de consumo sacrificadas que se incorporan en la producción, se puede usar el método de inventario perpetuo para construir una serie del acervo o stock de capital, expresada también en términos de canastas numéricas. Si uno analiza la producción usando la teoría del capital, no hay necesidad de dividir el aporte de capital en una contribución de calidad y cantidad como lo hacen los enfoque tradicionales de Solow y Kendrick, porque el capital representa un sacrificio de canastas de consumo o producción.

El análisis de la mano de obra bajo el TDA comienza tomando la factura salarial de un sector o de la economía. En cuentas nacionales, la información se denomina la compensación a los trabajadores y proviene de la llamada cuenta de generación del ingreso. Los datos de la masa salarial solo se obtienen para los sectores agregados y es igual a la suma del salario pagado a todas las clases de trabajo multiplicado por el número de horas de trabajo utilizadas en el sector. Al mismo tiempo, la masa salarial total es igual al salario básico ( $\left.\mathrm{W}^{*}\right)$ multiplicado por el número de unidades laborales básicas $\left(\mathrm{L}^{*}\right)$.

$$
\sum_{(i=1)}^{n} w_{i} L_{i}=W^{*} L^{*}
$$

Donde $w_{i}$ es el salario de cada una de las clases ocupacionales de un país y $L_{i}$ es la cantidad de trabajo contratado de cada clase ocupacional respectiva. Cabe recordar que el objetivo de agrupar el pago recibido por todos los trabajadores, en términos de unidades de trabajo básico, es lograr capturar dentro de la contribución del trabajo al crecimiento el aporte no solo de la cantidad de trabajadores, sino también la calidad de los mismos.

De hecho, $L^{*}$ es simplemente una medida expresada en unidades estándar o básicas de trabajo. Esa unidad representa a un trabajador con un nivel específico de habilidades de capital humano. Por ejemplo, en una planta industrial un conserje podría representar una unidad básica de trabajo, mientras que una secretaria podría ser equivalente a tres unidades laborales básicas, un ingeniero a diez, y el médico de la empresa podría ser equivalente a quince unidades laborales básicas.

$W^{*}$ es el salario de esta unidad básica de trabajo y sirve como deflactor de salarios. Más adelante se explican las consideraciones específicas necesarias para elegir este deflactor del salario laboral básico. Una vez que se define el salario básico, la cantidad de unidades laborales básicas es igual a:

$$
L^{*}=\sum_{(i=1)}^{n}\left(w_{i} L_{i}\right) / w^{*}
$$

Acá, $\sum_{(i=1)}^{n} w_{i} L_{i}$ (el gasto en salarios) es un número agregado; los datos desagregados no son necesarios cuando se utiliza la metodología del TDA. Con la cantidad de unidades laborales básicas, la masa salarial total se divide en dos componentes, la remuneración del trabajo básico y la remuneración del capital humano. Como puede observarse en la ecuación 3, el componente asociado al trabajo básico está relacionado simplemente con la cantidad de trabajadores $(L)$. Por su parte, el capital humano se mide como la diferencia entre la cantidad de unidades de trabajadores básicos y la cantidad real de trabajadores; en otras palabras, entre mayor capital humano tenga un trabajador, recibirá un mayor salario $y$, por tanto, representará una mayor cantidad de unidades básicas de trabajadores, por lo que la diferencia entre $L^{*} y L=1$ será más amplia en relación con un trabajador con menor nivel de capital humano.

Así, también, podrían existir trabajadores que tengan un nivel de capital humano negativo, comparado con el capital humano (salario) del trabajador básico. Se trata de trabajadores que 
ganarían por debajo de $w^{*}$. Sin embargo, como se utilizan son datos agregados, no se hace necesario preocuparse por una situación como la mencionada, pues la unidad de trabajo básica se debe elegir de forma que considere un trabajador con un nivel de capital humano muy elemental, por lo que lo trabajadores con niveles de capital humano negativo son más la excepción que la regla.

$$
\sum_{(i=1)}^{n} W_{i} L_{i}=\underbrace{w^{*} L}_{\text {Trabajo básico }}+\underbrace{W^{*}\left(L^{*}-L\right)}_{\text {Capital Humano }}
$$

$L$ es la cantidad de personal con un empleo en la economía y es información generalmente disponible por medio de las encuestas de hogares. Un aumento en el número de unidades laborales básicas de un período a otro cambiaría el total de la masa salarial en:

$$
W^{*} \Delta L^{*}=W^{*} \Delta L+W^{*} \Delta L^{*}-\Delta L
$$

Es decir, para un salario dado, la masa salarial aumenta debido a un incremento en el número de unidades laborales básicas o por las habilidades de capital humano.

El cambio en el capital humano en la ecuación 4 también puede dividirse en dos elementos: el componente de mantenimiento del capital humano (es decir, dotar a los nuevos trabajadores del capital humano promedio existente en el país) y el cambio en la calidad del capital humano. En este caso, el cambio en la calidad del capital humano se define como el incremento en la cantidad de unidades básicas de trabajo menos el capital humano promedio $\left(L^{*} / L\right)$ que se debe dotar a los nuevos trabajadores con el fin de que se incorporen sin afectar el nivel de capital humano promedio de la economía. Además, la suma del componente laboral básico de la ecuación 4 y el componente de mantenimiento del capital humano es igual a la contribución tradicional del factor trabajo al cambio de producción de los modelos de Solow y Kendrick. Esto demuestra que los modelos tradicionales subestiman la contribución de los trabajadores al crecimiento, cuando existen mejoras en el capital humano, con la consecuente sobreestimación de la PTF, pues esta se obtiene como un residuo.

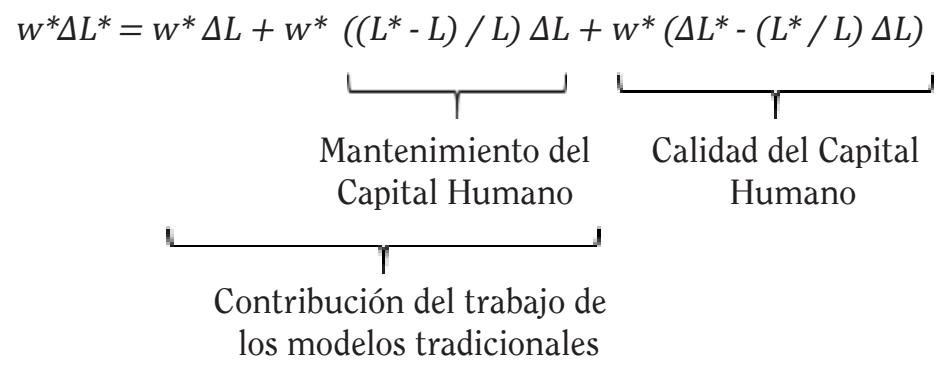

Por lo tanto, el enfoque tradicional de las fuentes del crecimiento económico de Solow y Kendrick, el cual es seguido por autores como Jorgenson et al. (1987) y Jorgenson (1995a, 1995b), entre muchos otros ${ }^{3}$, no considera los cambios en la calidad del capital humano como parte de la contribución del aporte laboral. El residuo del enfoque tradicional será mayor si hay una mejora en la calidad del capital humano durante el período, ya que esta se destina al residuo según el enfoque tradicional. A diferencia del enfoque tradicional, la incorporación de $L^{*}$ permite identificar todas las contribuciones del capital humano al crecimiento $y$, por lo tanto, elimina todos los aspectos laborales del residuo.

3 Por ejemplo, Sickles y Zelenyuk (2019) hacen una revisión extensa de las diferentes metodologías para estimar la productividad $y$ las ventajas y desventajas de cada una de ellas. 
Para estimar el residuo bajo la metodología del TDA en este documento, el valor real del PIB se expresa primero en cestas de producción mediante el uso del Deflactor implícito del PIB. El valor real del PIB se puede dividir en dos componentes: el ingreso laboral (la masa salarial) y el ingreso de la propiedad. Una forma alternativa de representar el ingreso de la propiedad es multiplicando el stock de capital por la tasa de rendimiento del capital bruto de la depreciación.

$$
Y=W^{*} L^{*}+\left(r^{*}+\delta^{*}\right) K^{*}
$$

Donde $Y$ es el valor real del PIB, lo cual, a nivel nacional, es igual al quantum de la producción. $K^{*}, r^{*} y \delta^{*}$ son el stock de capital, la tasa de rendimiento y la tasa de depreciación de la serie de stock de capital formada utilizando el TDA. Teniendo en cuenta la ecuación 6 , el PIB real puede crecer a partir de tres fuentes, donde $\mathrm{R}^{*}$ se interpreta como la PTF, pues es el cambio en la producción que no está explicado por cambios en el uso de los insumos trabajo y capital.

$$
Y=W^{*} \Delta L^{*}+\left(r^{*}+\delta^{*}\right) \Delta \mathrm{K}^{*}+\mathrm{R}^{*}
$$

Por lo tanto, la ecuación para el residuo de la metodología del TDA como un porcentaje del valor real de la producción es,

ó,

$$
\mathrm{R}^{*} / \mathrm{Y}=\Delta \mathrm{Y} / \mathrm{Y}-\mathrm{W}^{*}\left(\Delta \mathrm{L}^{*}\right) / \mathrm{Y}+\left(\mathrm{r}^{*}+\delta^{*}\right)\left(\Delta \mathrm{K}^{*}\right) / \mathrm{Y}
$$

$$
\mathrm{R}^{*} / \mathrm{Y}=\Delta \mathrm{Y} / \mathrm{Y}-\left(\left(\mathrm{w}^{*} \mathrm{~L}^{*}\right) / \mathrm{Y}\right)\left(\Delta \mathrm{L}^{*}\right) / \mathrm{L}^{*}+\left[\left(\left(\mathrm{r}^{*}+\delta^{*}\right) \mathrm{K}^{*}\right) / \mathrm{Y}\right]\left(\Delta \mathrm{K}^{*}\right) / \mathrm{K}^{*}
$$

El uso de $L^{*}$ permite incluir cada pequeña contribución de todo tipo de clases de mano de obra sin especificar explícitamente miles de clases de mano de obra, sus salarios y horas trabajadas. Así, se obtiene un mejor resultado con una menor cantidad de datos y menos esfuerzo. Sin embargo, hay que recordar que para que la metodología brinde resultados robustos es necesario definir un salario adecuado para la unidad básica de trabajo $\left(w^{*}\right)$.

La ecuación 9 difiere de la siguiente ecuación 10 del enfoque tradicional de contabilidad de crecimiento en dos aspectos, donde $\mathrm{S}_{\mathrm{L}}$ y $\mathrm{S}_{\mathrm{K}}$ se refieren a la porción del valor de la producción que son pagadas a los trabajadores $y$ a los dueños del capital, en ese orden.

$$
\Delta Q / Q=S_{L} \Delta L / L+S_{K} \Delta K / K+R / Q
$$

En primer lugar, hay una subestimación de la contribución del trabajo al crecimiento, lo cual produce a su vez una sobreestimación de la productividad. En otras palabras, no toda la calidad del trabajo se asigna a la contribución del trabajo, como sí ocurre en la ecuación 9. En segundo lugar, existe un efecto de precio relativo, porque la ecuación 9 es el residuo expresado en términos del valor del producto deflactado por un índice de precios agregado, mientras que en la ecuación 10 el residuo está en términos de quantum o cantidades. A nivel nacional, la única diferencia entre las dos ecuaciones es el efecto de la calidad del trabajo.

A diferencia del enfoque tradicional, la PTF ahora se puede interpretar como innovaciones de reducción de costo real y no solo como un residuo de factores inexplicados que afectan la producción. En las palabras de Harberger (1993),

Las reducciones en la prima de habilidades promedio están implícitamente consideradas como un elemento adicional de reducción de costos. Por lo tanto, cuando disminuya cualquier prima de habilidades dada, la contribución al crecimiento del 
componente de cambio de calidad se reducirá, generando un mayor residuo atribuido al crecimiento de la PTF. Pero recordemos que la reducción de la prima de habilidades para, digamos, ingenieros es una reducción de costos en lo que respecta a las empresas que usan esas habilidades. (p. 11)

\section{DATOS UTILIZADOS}

Para estimar el crecimiento de la productividad utilizando el TDA de contabilidad de crecimiento, es necesario partir de la cuenta de generación de ingreso de las cuentas nacionales de producto interno bruto. Esta cuenta divide el PIB en remuneración a asalariados, impuestos netos a la producción e importaciones y el llamado excedente de explotación que refleja las utilidades de los dueños de los insumos de capital. Esta información proviene de la matriz insumo - producto del BCCR $-y$ el cálculo fue incluido en las cuentas nacionales hasta el año 2013. Luego de ese año, se tomó la evolución del pago a asalariados con respecto al PIB para proyectar esa proporción hasta el año 2019.

En segundo lugar, se debe elegir el primer deflactor para expresar todas las variables en unidades reales. Luego se debe elegir el segundo deflactor para obtener la cantidad de unidades básicas de trabajo, a partir de la remuneración de los asalariados. Por último, se requiere obtener de las cifras del PIB, la formación bruta de capital con el fin de crear una serie del acervo o stock de capital.

\subsection{La elección del primer deflactor}

El primer deflactor utilizado en el TDA es un índice de precios para expresar todas las variables en canastas de consumo o de producción. Existen dos candidatos: el índice de precios al consumidor (IPC) y el deflactor implícito del PIB (IPIB).

La elección del índice va a depender de la disponibilidad y consistencia de la información. Como el presente estudio se está realizando para el periodo 1960-2019, se decidió utilizar el IPIB pues el IPC ha cambiado su metodología en múltiples ocasiones a lo largo de este periodo, incluso en más ocasiones que el cambio de base que ha experimentado la metodología de cálculo del PIB. Además, la información del IPIB es más consistente para los primeros años que incluye el presente estudio.

A pesar de lo anterior, como puede observarse en el gráfico 1, la evolución de estos dos índices ha sido muy similar en estos 60 años. Esto significa que ambos índices estarían funcionando de manera similar. 


\section{GRÁFICO 1 \\ EVOLUCIÓN DEL ÍNDICE IMPLÍCITO DEL PIB Y DEL ÍNDICE DE PRECIOS AL CONSUMIDOR $(2000=100$}

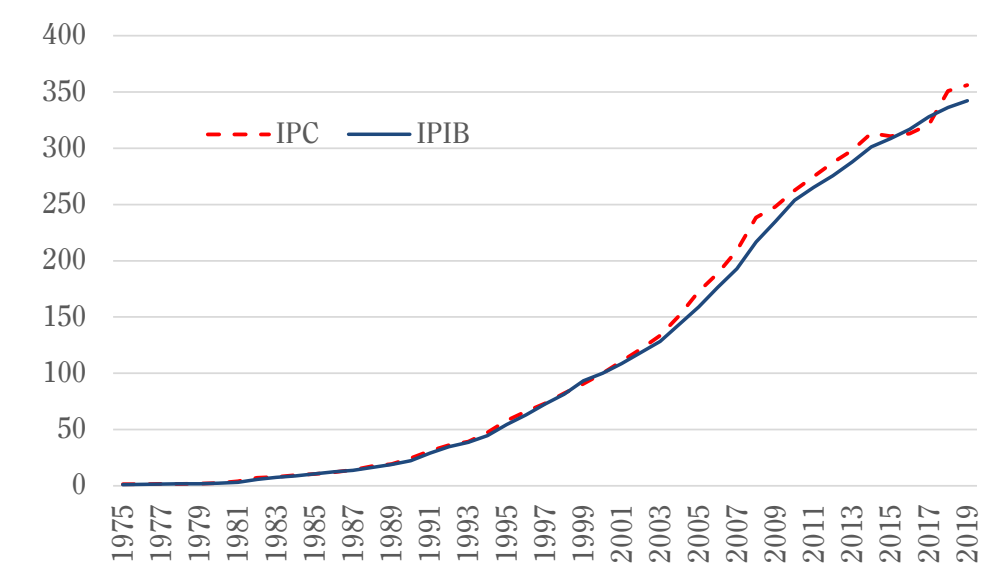

Fuente: Elaboración propia con información del BCCR.

La mayoría de los estudios de contabilidad de crecimiento utilizan el IPIB como deflactor, lo cual le agrega un atractivo adicional para efectos de comparabilidad. En otros países, también suele ser más conveniente usar el IPIB, pues el IPC se ve afectado por ciclos políticos durante periodos cortos, en especial al precio de los bienes regulados. Esto produce que las variables reales exhiban una alta volatilidad de un año a otro. Sin embargo, para largos periodos, la elección de cualquiera de estos índices no debería tener importancia, pues los años con crecimientos de IPC artificialmente bajos son compensados con crecimientos elevados del IPC en los años subsiguientes.

El uso de un solo índice para expresar todas las variables en términos reales tiene su fundamento en la teoría del capital y la evaluación de proyectos. La manera correcta de analizar la inversión en un proyecto es expresar tanto los costos como los beneficios en las mismas unidades reales. O sea, tanto la inversión como la corriente futura de ingreso son deflactadas por el mismo índice de precios.

Lo mismo aplica para la teoría del capital. Un inversionista piensa en término de unidades sacrificadas de consumo y no, por ejemplo, en términos de unidades de maquinaria puestas en servicio. Por lo tanto, no es solo conveniente sino también más atractivo trabajar con un único índice para obtener valores reales.

\subsection{La construcción del acervo de capital}

Una vez que las variables se expresan en términos reales, el acervo de capital se puede estimar utilizando el método de inventario perpetuo. Este asegura que la contribución del stock de capital al crecimiento del PIB es proporcional al stock de capital en sí. La formación bruta de capital se obtiene del cálculo del PIB del Banco Central y el stock de capital al final de un año dado es igual a,

$$
K_{t}=(1-\delta) K_{t-1}+I_{t}
$$

Donde $I_{t}$ es la formación bruta de capital y $\delta$ es la tasa de depreciación del capital. Por lo tanto, el stock de capital al final del período es igual al stock de capital al inicio del período menos la depreciación más la inversión. 
En la serie del PIB con año base 1991, los datos de las cuentas nacionales de ingresos y productos distinguen tres clases de insumos de capital en la economía: inversión en edificaciones, inversión en maquinaria y equipo, y cambio en los inventarios de los productores que es un activo no depreciable. De esta forma, la estimación del stock de capital incorpora las diferencias entre estos activos.

Además, se asume una tasa de depreciación anual del 8\% para maquinaria y equipo, y del 2.5\% para edificaciones. Estas mismas tasas fueron utilizadas por Harberger (1993) y Robles Cordero $(2000)^{4}$ y se supone que representan promedios que incluyen el deterioro físico y la obsolescencia. Estas tasas de depreciación también caen dentro del rango de las tasas utilizadas por Jorgenson et al. (1987) y Jorgenson (1995a, 1995b), entre otros, e implican que la inversión en maquinaria y equipo perderá el $34 \%$ de su productividad, después de cinco años, y el $81 \%$, después de veinte años. Mientras tanto, la inversión en edificaciones perderá el $12 \%$ de su productividad, después de cinco años, y el 30\%, después de veinte años. En el caso de la serie del PIB con el año base 2012, las cuentas nacionales incluyen una estimación de la depreciación, por lo que se utiliza dicha serie en los cálculos del acervo de capital.

Lo último que se necesita para construir la serie de stock de capital es el nivel del período inicial. Aquí, la idea es encontrar un período lo más lejano posible en el tiempo, porque el nivel inicial del stock de capital perderá importancia después de, por ejemplo, veinte años; ya que la mayor parte se habrá depreciado, y su importancia relativa es menor en comparación con la acumulación de nuevas inversiones.

Para encontrar el stock de capital inicial, se puede suponer que en el pasado el stock de capital creció a la misma tasa que el PIB real (g). Así que,

$$
\mathrm{K}_{\mathrm{t}} / \mathrm{K}_{(\mathrm{t}-1)}=\mathrm{g}, \forall \mathrm{t} \leq 0
$$

Al utilizar las ecuaciones 11 y 12, se obtiene que la inversión en el periodo 1 es igual a:

$$
I_{1}=K_{1}-(1-\delta) K_{0}=g K_{0}+\delta K_{0}=(g+\delta) K_{0}
$$

Por lo que,

$$
\mathrm{K}_{0}=\mathrm{I}_{1} /(\mathrm{g}+\delta)
$$

En consecuencia, para estimar el stock inicial de capital para edificaciones y maquinaria y equipo, se necesita encontrar un período de crecimiento de producción "estable" (g) tan distante como sea posible, $y$ aplicar la ecuación 14 a la inversión promedio durante ese periodo. Para el stock de capital de inventarios, se supone que el stock de capital inicial es igual al PIB del año inicial multiplicado por la razón del cambio acumulativo en los inventarios, sobre el cambio acumulado en el PIB, durante los años para los cuales hay datos disponibles. El stock de capital total para la economía es igual a la suma de los tres tipos de insumos de capital.

Una vez construida la serie de stock de capital, las tasas de rendimiento y de depreciación total necesarias en la ecuación 9 se calcularon utilizando los datos de excedentes de explotación de la cuenta de generación de ingreso del PIB de cuentas nacionales.

\footnotetext{
4 Estas tasas fueron utilizadas por estos autores para todos los países de América Latina, los Estados Unidos y a nivel de industrias con un nivel de desagregación a 4 dígitos de la categoría CIIU.
} 


\subsection{La elección del segundo deflactor}

El segundo deflactor de la metodología del TDA está relacionado con la definición de la unidad de trabajo básica. Si se es capaz de elegir el deflactor de salario adecuado ( $\left.\mathrm{W}^{*}\right)$, entonces es posible capturar la contribución completa de la calidad del capital humano como parte de la contribución del trabajo al crecimiento y no como parte de la PTF.

La elección del deflactor de salarios debe reflejar las características de calidad o las habilidades de capital humano de la unidad de trabajo básica. En otras palabras, si el salario del trabajador con habilidades laborales básicas está cambiando a lo largo del tiempo, entonces el índice elegido debe reflejar esos cambios.

Hay varias opciones para este deflactor. Una es tomar dos tercios del PIB per cápita como deflactor. De acuerdo con Harberger (1993), este es conveniente por tres razones:

a) En casi todos los países se encontrará un número significativo de trabajadores cuyas ganancias a tiempo completo son alrededor de $2 / 3$ de un PIB per cápita de ese país,

b) en todos los casos, tales trabajadores tienden a estar muy al extremo inferior del espectro de la calidad del trabajo, justificando así nuestra atribución de $\mathrm{W}^{*}$ como aplicable al "trabajo básico";

c) la volatilidad de esta medida es muy baja en comparación con las series competidoras de ocupaciones específicas. (p. 15)

Hay otras opciones para el deflactor del trabajo básico. Por ejemplo, se puede utilizar una serie de salarios de trabajadores del sector primario (agricultura, ganadería y pesca), pensando que este sector en Costa Rica ha sido relativamente no regulado y no hay sindicatos que afecten significativamente los salarios. Además, los trabajadores en el sector primario no están altamente calificados y la calidad de esas habilidades ha sido relativamente estable a través del tiempo. Sin embargo, tal como lo indica Harberger (1993), dicho índice está influenciado por factores que afectan a la actividad específica, como los cambios en las políticas proteccionistas. Por lo tanto, esta serie de salarios será más volátil y las unidades laborales básicas mostrarán oscilaciones no relacionadas con los cambios en las habilidades de los trabajadores, lo que hace que esta elección del deflactor sea inapropiada. Otra desventaja es tener una serie suficientemente larga para una clase ocupacional específica de trabajador básico. Esto se complica aun más cuando nos ubicamos en los años sesenta y notamos que las encuestas de hogares no eran frecuentes y que, desde esa época, han existido cambios metodológicos que hacen imposible la comparación, lo cual genera sesgos innecesarios a las estimaciones de la PTF.

En el gráfico 2 se traza un índice de $2 / 3$ del PIB per cápita y un índice de salarios reales en el sector primario, utilizando la información de la Encuesta Continua de Empleo. Se puede observar que el índice salarial del sector primario es más volátil y sus oscilaciones son más pronunciadas que en el caso del 2/3 del índice del PIB per cápita. Además, el crecimiento de los salarios reales en el sector primario es considerablemente menor que el crecimiento de $2 / 3$ del PIB per cápita. Por último, es prácticamente imposible construir una serie consistente de salarios en el sector primario para toda la muestra considerada en el presente estudio. 


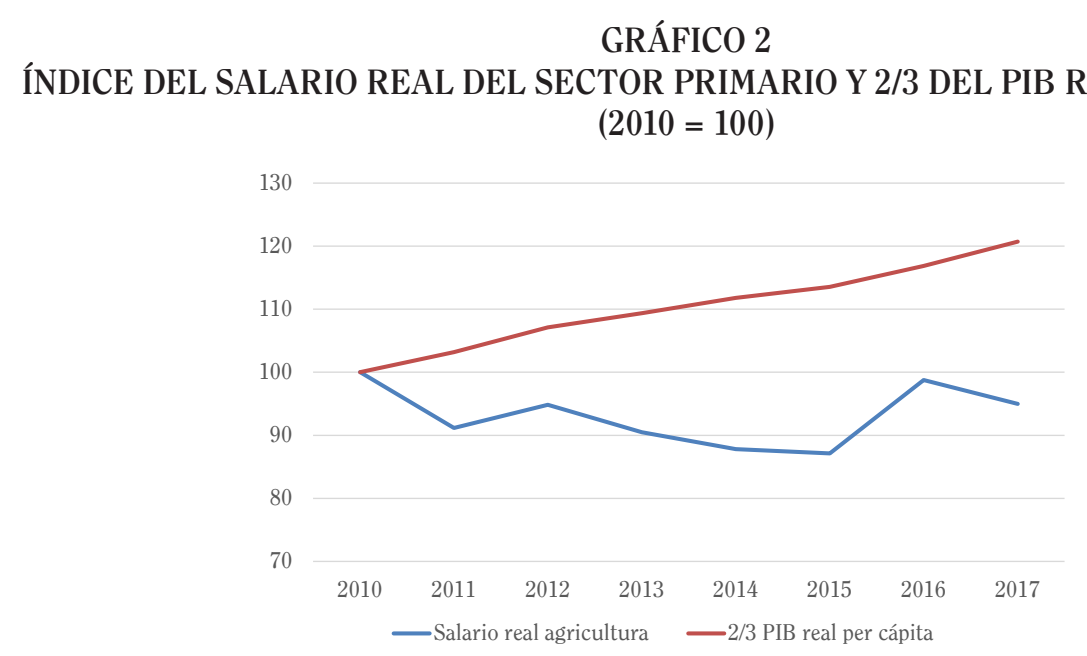

Fuente: Elaboración propia con información del BCCR y del INEC.

Debe mencionarse que la proporción del PIB per cápita (dos tercios en este caso) elegida como índice de trabajo básico solo es relevante para dividir la contribución del trabajo entre la contribución del trabajo básico y del capital humano al crecimiento. La misma contribución total del trabajo se obtendría si $W^{*}=\lambda(G D P / N)$, ya que en la ecuación 8 se consideran los cambios de $L^{*}$. Por lo tanto, mientras se mantenga la contribución laboral al crecimiento, es importante elegir el índice con la tendencia correcta del salario de la unidad de trabajo básica.

En la siguiente sección se utiliza la cantidad de personas empleadas a partir de la información provista por el INEC como definición de aporte laboral para la metodología del TDA. Como se mencionó anteriormente, esta solo afectaría la división entre la cantidad y las contribuciones de calidad del trabajo según el TDA.

Finalmente, la compensación nominal a los empleados proviene de la cuenta de generación de ingreso del PIB, como se mencionó en la sección anterior. Los impuestos indirectos son parte del valor agregado $y$ no deben considerarse ingresos de la propiedad únicamente porque tanto los insumos de capital como la mano de obra contribuyen al producto. Así, una proporción (la parte del trabajo) de los impuestos indirectos se atribuye a los ingresos laborales según la TDA.

Como se ha indicado en este artículo, numerosos autores han concluido que existe una correlación importante entre la tasa de crecimiento de la economía y el crecimiento de la productividad $^{5}$. Más aun, los países que experimentan una mayor tasa de crecimiento de la producción lo hacen porque su productividad crece más rápidamente.

Lo mismo ocurre dentro de un país. El crecimiento es más elevado en aquellos años en donde la productividad crece más rápidamente. El gráfico 3 presenta la relación entre el cambio en la tasa de crecimiento del PIB y el cambio en el crecimiento de la productividad desde 1960 hasta el 2019, a partir de las estimaciones realizadas de acuerdo con la ecuación 9. En este gráfico, puede observarse que la relación es positiva y cercana al 69 \%. O sea, más de dos terceras partes del cambio en la tasa de 


\section{GRÁFICO 3 \\ CAMBIOS EN EL CRECIMIENTO DEL PIB E INCREMENTO EN LA PTF EN COSTA RICA: 1960-2019 (EN PORCENTAJES)}

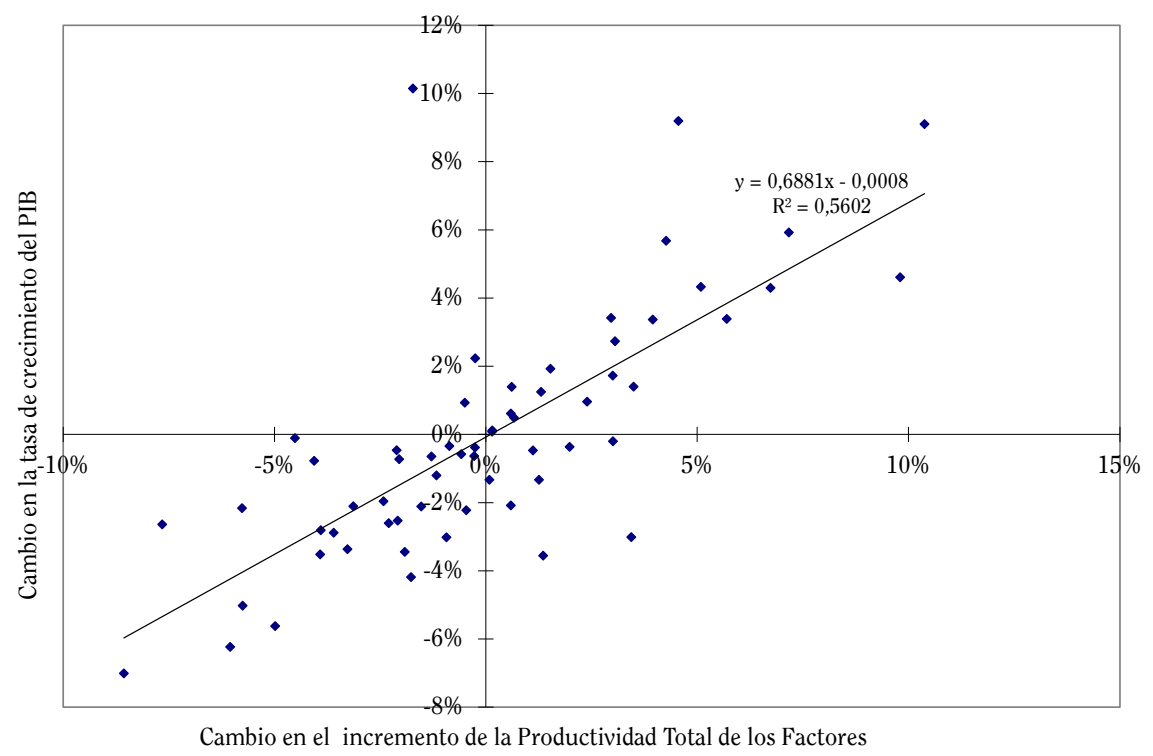

Fuente: Elaboración propia

crecimiento del PIB es explicado por cambios en la tasa de crecimiento de la productividad. El restante $31 \%$ se explica por cambios en las contribuciones de los factores de producción (trabajo y capital).

El gráfico 4 muestra las fuentes del crecimiento económico para Costa Rica entre 1960 y 2019, según la ecuación 9. La información se presenta por décadas, con excepción de los años ochenta que se divide en lustros, dada la crisis de la primera mitad de esa década. Las cifras indican que el crecimiento en la productividad, como fuente de contribución al crecimiento económico, ha sido volátil durante el periodo estudiado, cuando se compara con las contribuciones de los insumos de capital y trabajo. Durante el periodo analizado, el $14 \%$ del crecimiento económico es explicado por el crecimiento de la PTF. En el caso del trabajo y el capital, su contribución al crecimiento siempre ha sido positiva, mientras que la productividad no logró crecer durante la crisis de la deuda de los años ochenta y en los dos mil, a raíz de los efectos de la crisis llamada "dot com" al inicio de esa década, la Gran Crisis Financiera internacional del 2008 y 2009; y en los años 2018 y 2019, por el crecimiento desproporcionado del déficit fiscal.

El otro elemento destacable es que las contribuciones del trabajo y del capital al crecimiento fueron más fuertes durante las décadas de los sesenta y setenta. Esto provocó que la tasa de crecimiento de la producción fuera mayor en comparación con los periodos posteriores. Durante esos años, la economía costarricense se basaba en un modelo de sustitución de importaciones. Sin embargo, cuando los países basan su crecimiento en acumulación de factores y no en productividad, la economía se ve afectada por las productividades marginales decrecientes de estos insumos y el crecimiento económico potencial decae en el tiempo. El agotamiento del modelo de sustitución de importaciones se vuelve evidente con la explosión de la crisis de la deuda, cuando el crecimiento económico se estanca a inicios de los años ochenta, a raíz de una caída en la productividad de la economía. Luego el crecimiento de la productividad se reactiva con el modelo de promoción de 


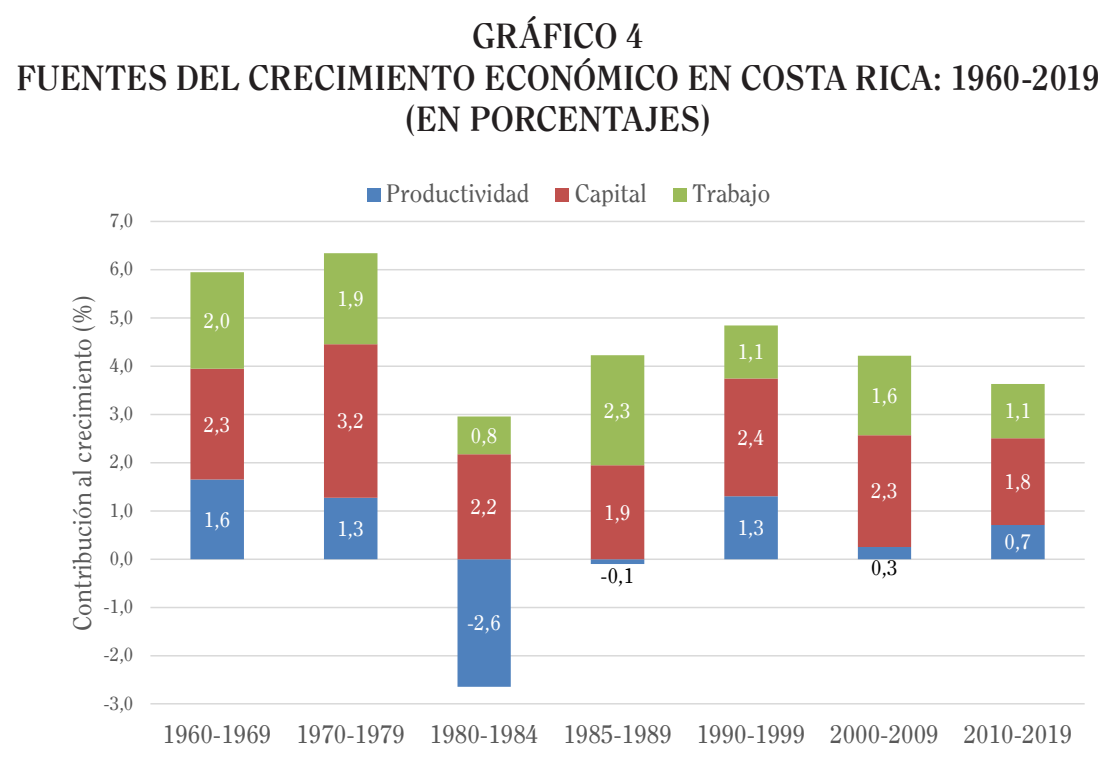

Fuente: Elaboración propia

exportaciones, para pasar luego al modelo de atracción de inversión extranjera directo a partir del año 1997 con la llegada de la empresa Intel a Costa Rica.

La ventaja principal de la metodología del TDA utilizada en el presente artículo para hallar las fuentes de crecimiento económico es que permite desagregar la contribución del trabajo en unidades básicas de trabajo, el mantenimiento del capital humano de los trabajadores y la mejora en la calidad de estos, tal y como fue explicado en la ecuación 5. Para hacer esto, es necesario contar con estadísticas de empleo, las cuales se producen de forma sistemática en Costa Rica desde el año 1977.

GRÁFICO 5

COSTA RICA: DESCOMPOSICIÓN DE LA CONTRIBUCIÓN DE LA MANO DE OBRA AL CRECIMIENTO 1977-2019 (EN PORCENTAJES)

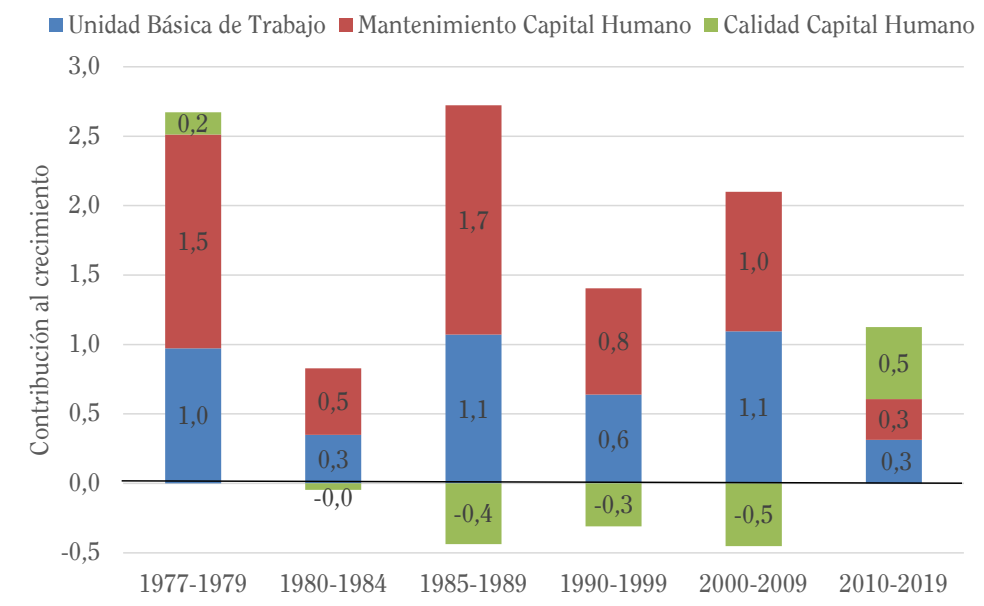

Fuente: Elaboración propia 
El gráfico 5 presenta la forma en la contribución de la mano de obra al crecimiento económico se descompone en estos tres elementos.

El elemento más notable de esta descomposición de la contribución del factor trabajo al crecimiento económico es que la mano de obra empleada no ha tenido mejoras sustanciales en su calidad con excepción del último periodo de análisis . En este periodo, la economía costarricense se ha caracterizado por un crecimiento muy incipiente en el empleo. O sea, es un periodo en donde la tasa de desempleo ha aumentado por encima del 12 \% y la tasa de participación de la población económicamente activa ha decrecido. Estas cifras sugieren que las personas que se mantuvieron laborando o encontraron empleo presentaban los niveles más elevados de capital humano, lo que ocasionó que el nivel promedio de capital humano de las personas empleadas creciera en la última década y con ello su contribución al crecimiento económico.

Por el contrario, en las décadas previas y desde los años ochenta, los trabajadores que engrosaron la fuerza de trabajo traían un nivel de capital humano bajo en relación al promedio. Esta situación constituyó un freno de la contribución del trabajo al crecimiento económico.

\section{GRÁFICO 6 \\ COSTA RICA: ÍNDICE DE PRODUCTIVIDAD TOTAL DE LOS FACTORES 1960-2019 $(1960=100)$}

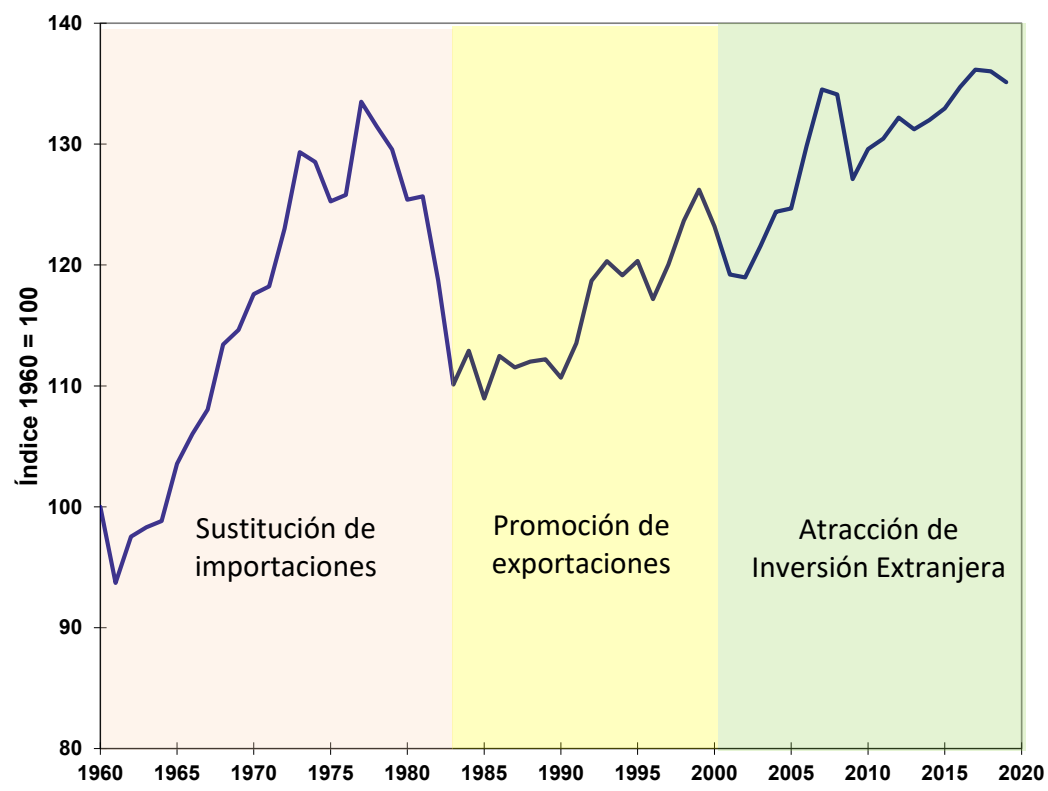

Fuente: Elaboración propia

La estimación de la evolución de la productividad para Costa Rica desde el año 1960 se presenta en el gráfico 6. Como puede observase, el periodo analizado se divide de acuerdo al modelo de crecimiento económico seguido por el país.

Es claro en el gráfico 6, el agotamiento del crecimiento en la productividad del primer periodo de sustitución de importaciones, pues la productividad empezó a decrecer a partir del año 1978, incluso antes de la crisis de la deuda (1981 en adelante). Además, es notable que durante la época en la cual el país presentó la mayor inestabilidad macroeconómica en su historia reciente (1980-1990), también coincidió con el mayor descenso y posterior estancamiento de la PTF. Una vez 
que las variables macroeconómicas se estabilizaron, de los años noventa en adelante, la PTF vuelve a crecer.

Los ochenta fueron una década perdida en términos de crecimiento de productividad. Estos fueron años caracterizados por el ajuste económico y la adopción de un modelo de promoción de exportaciones basada fuertemente en incentivos fiscales. Es a partir de 1990 que la economía costarricense vuelve a mostrar incrementos en productividad. De hecho, el nivel de productividad que tenía la economía en 1977 no se alcanza de vuelta hasta más de 30 años después, en 2007, cuando el país pasa a basar su crecimiento en un modelo de promoción de inversión extranjera directa. A partir de allí, el crecimiento en la productividad enfrenta una nueva caída debido a la Gran Crisis Financiera internacional de finales de la década de los 2000, para luego retornar a una senda de crecimiento, la cual seguramente volverá a estancarse a partir del año 2020, luego de la pandemia mundial por Coronavirus. En síntesis, el nivel de productividad de la economía costarricense en el año 2020 será inferior al mostrado durante la década de los setenta, lo cual implica más de 40 años de estancamiento de la productividad. Esto es preocupante, pues la productividad es el principal elemento que explica las diferencias de crecimiento económico entre países.

\section{Efecto de la estabilidad macroeconómica en el crecimiento de la productividad en Costa Rica}

Como se ha indicado en secciones previas, existe una infinidad de factores que inciden en la productividad total de los factores de un país, esta entendida como una reducción de costos reales. A pesar de ello, diversos estudios muestran la posibilidad de influir en el crecimiento de la productividad mediante el diseño de políticas públicas.

Por ejemplo, Kim y Loayza (2017) muestran para un grupo de 65 países que existe una asociación positiva entre el crecimiento de la productividad y la infraestructura, el nivel de educación, la eficiencia en los mercados, la innovación y la institucionalidad del país.

Otros autores como Berument et al. (2011) han encontrado efectos positivos sobre la PTF derivados de condiciones macroeconómicas más favorables, como la estabilidad de la inflación, la apertura al comercio internacional (probablemente como una proxy de innovación tecnológica por la importación de nuevas tecnologías) y la profundización del mercado financiero.

Straub (2008) presenta evidencia para 140 países sobre el efecto positivo de la inversión en infraestructura sobre el crecimiento económico. Aschauer (1989) también indica que la inversión de los Estados Unidos en infraestructura pública fue crucial en el crecimiento de la productividad de ese país en el tercer tercio del siglo pasado.

Los efectos de la educación sobre la productividad y el crecimiento han sido ampliamente estudiados. Por ejemplo, Barro (2001) mostró una relación positiva en más de 100 países entre el crecimiento de la productividad y la calidad de la educación de los alumnos varones. Otros como Erosa et al. (2010) y Bronzini y Piselli (2009) han encontrado un efecto positivo de una mayor escolaridad sobre la PTF.

La innovación se puede medir por medio de diferentes proxys, dada la generalidad del término. Una de ellas es la inversión extranjera directa (IED), como una forma de adopción de nuevas tecnologías. Por ejemplo, Alfaro et al. (2009) y Chang et al. (2009) muestran efectos positivos de la IED sobre el crecimiento económico, aunque la IED debe combinarse con políticas educativas e institucionales para que los países puedan adoptar las nuevas tecnologías. Lo mismo sucede con los efectos de la apertura comercial, pues autores como Coe et al. (1997) y Maiti (2013) mostraron que el aumento en los flujos de comercio de países seleccionados ha tenido un impacto positivo sobre la productividad.

El propósito de esta sección del documento es evaluar el papel de la inestabilidad macroeconómica en el crecimiento de TFP, mediante el uso de un análisis cuantitativo paramétrico. 
Basado en la disponibilidad de datos para diversas variables, se eligió explorar las volatilidades de tres variables como posibles indicadores de inestabilidad macroeconómica: inflación, apertura de una economía y profundidad de los mercados financieros.

Es importante tener en cuenta que las medidas de volatilidad de las tres variables que se utilizaron provienen de una especificación de forma reducida. Por lo tanto, se debe hacer una advertencia sobre la interpretación de los coeficientes estimados de las variables de volatilidad para las especificaciones. Los coeficientes estimados evalúan cómo las volatilidades en las tres variables, como medida de inestabilidad macroeconómica, afectan el crecimiento de la PTF. Estos no capturan los choques estructurales que pueden influir en estas tres variables, después de que se toman en cuenta otros factores las afectan.

PTF e Inflación. La volatilidad de la inflación es la primera medida de inestabilidad macroeconómica que se considera dentro de las estimaciones realizadas. La volatilidad de la inflación afecta negativamente la eficiencia en la asignación de los recursos, aumentando el desempleo y disminuyendo el crecimiento (Friedman, 1977). Específicamente, la incertidumbre de la inflación obstaculiza la eficiencia distributiva del sistema de precios. Friedman argumenta que los cambios no anticipados en la inflación causarán errores sistemáticos de percepción por parte de los empleadores y empleados que inicialmente provocará que el desempleo se desvíe de su tasa natural.

Además, la incertidumbre de la inflación puede ofuscar la distinción entre choques reales y nominales que los agentes económicos suponen responderán diferentemente (Lucas, 1973). La evidencia empírica respalda esta hipótesis (Froyen \& Waud, 1987; Hafer, 1986; Holland, 1986). Asimismo, se ha mostrado que la incertidumbre sobre la inflación incrementa el ahorro por precaución, debido a la menor producción y esto disminuye las tasas de interés nominales (Dotsey \& Sarte, 2000). La variabilidad de la inflación aumenta el ahorro y crea el incentivo para flexibilizar la política monetaria y, así, disminuir las tasas de interés, lo cual estimula la inversión. Si la nueva inversión tiende a aumentar el stock de capital que usa tecnologías más avanzadas, aumentará la PTF (Cukierman \& Meltzer, 1986; Hahn, 1970; Juster \& Taylor, 1975; Juster \& Wachtel, 1972a, 1972b).

PTF y apertura comercial. La volatilidad de la apertura capta la capacidad de la economía para proporcionar materias primas importadas para el proceso de producción, así como maquinaria y equipo con fines de inversión y equipos de repuesto para el stock de capital existente. Una mayor variabilidad de apertura puede disuadir a las empresas de adoptar tecnologías extranjeras más eficientes, debido a las dificultades que podrían experimentar en el futuro con respecto a las piezas de repuesto o los requisitos de entrada; en su lugar, adoptan tecnologías más bajas disponibles a nivel nacional y esto disminuye la PTF. Rodrik (1998) desarrolla un modelo macroeconómico donde un aumento en el riesgo de las exportaciones requiere una reasignación de los recursos de la economía hacia la actividad segura, incluso cuando el retorno a las actividades más seguras está por debajo del retorno (medio) de otras actividades. Esto finalmente disminuye el crecimiento de la PTF. Montalbano et al. (2005) proporcionan evidencia empírica de que la vulnerabilidad comercial en la década de 1990 afectó adversamente el bienestar de los países de Europa del Este.

PTF y profundidad financiera. La volatilidad de la profundidad de los mercados financieros mide la inestabilidad en el sector financiero. La percepción de una mayor vulnerabilidad afecta el comportamiento de los intermediarios financieros, así como de las empresas que pueden acceder el crédito. Para ser específicos, una mayor vulnerabilidad en el sistema financiero desalienta a los intermediarios financieros a otorgar préstamos a largo plazo, a pesar de que hacerlo podría mejorar la PTF, y tienden a concentrarse en dar préstamos a corto plazo. Las empresas también están menos dispuestas a recibir crédito de los intermediarios financieros, lo que reduce las nuevas inversiones. Además, en tiempos de mayor vulnerabilidad (o la percepción de ello), las compañías tienden a usar recursos internos para financiar sus inversiones. La menor financiación externa también puede sugerir que sin intermediarios financieros, las inversiones se asignan de manera menos eficiente. Por lo tanto, la mejora de la productividad es menor en un nivel dado de inversión. 
Los mercados incompletos, asociados con la falta de intermediación financiera, reducen la PTF al desplazar recursos de proyectos más riesgosos, pero también más productivos (Angeletos, 2006). Sin embargo, la intermediación financiera aumenta la composición cualitativa de la inversión más que la cantidad de inversión, y esto mejora la PTF (Evers et al., 2008). El modelo que vincula el desarrollo financiero con el crecimiento económico (Acemoglu \& Zilibotti, 1997) ha sido respaldado por la evidencia empírica (Levine et al., 2000).

\subsection{Modelo cuantitativo}

Para llevar a cabo el análisis cuantitativo, se utilizan las series tiempo de la PTF, calculada como se describió en la primera parte de este artículo, de la inflación, de la apertura comercial medida como el cociente de importaciones más exportaciones a PIB, de la inversión extranjera directa a PIB y de la profundidad financiera representada por la razón de M1 o M2 a PIB.

Como se muestra en el cuadro 1, para evaluar en qué forma estas variables entran en el análisis, se realizó una prueba de raíz unitaria. Si estas series tienen una raíz unitaria y entran en los análisis en niveles, entonces los análisis econométricos podrían dar estimaciones espurias. La CUADRO 1

PRUEBA SOBRE RAÍZ UNITARIA (TEST DE ADF)

\begin{tabular}{|c|c|c|c|c|}
\hline Variable & Tipo & $\begin{array}{c}\text { Componentes } \\
\text { prueba }\end{array}$ & Rezagos & $\mathrm{ADF}$ \\
\hline \multirow[t]{3}{*}{ Productividad total de los factores } & Niveles & Constante & 3 & -0.814 \\
\hline & Niveles & Constante y tendencia & 3 & -1.68 \\
\hline & Pimeras diferencias & Constante & 2 & $-4.227 *$ \\
\hline \multirow[t]{3}{*}{ Inflación } & Niveles & Constante & 3 & -1.178 \\
\hline & Niveles & Constante $y$ tendencia & 3 & -0.541 \\
\hline & Pimeras diferencias & Constante & 2 & $-4.029 *$ \\
\hline \multirow[t]{3}{*}{ Apertura Comercial(X+M/PB) } & Niveles & Constante & 3 & -1.819 \\
\hline & Niveles & Constante $y$ tendencia & 3 & -1.099 \\
\hline & Pimeras diferencias & Constante & 2 & $-6.358-$ \\
\hline \multirow[t]{3}{*}{ IED (Inversión externa directa / PIB) } & Niveles & Constante & 3 & -0.97 \\
\hline & Niveles & Constante y tendencia & 3 & -2.246 \\
\hline & Pimeras diferencias & Constante & 2 & $-6.869-$ \\
\hline \multirow[t]{3}{*}{ Profundidad Financiera (M2 / PIB) } & Niveles & Constante & 3 & -0.289 \\
\hline & Niveles & Constante $y$ tendencia & 3 & -2.171 \\
\hline & Pimeras diferencias & Constante & 2 & $-4.210^{*}-$ \\
\hline \multirow[t]{3}{*}{ Profundidad Financiera (M1 / PIB) } & Niveles & Constante & 3 & -0.846 \\
\hline & Niveles & Constante y tendencia & 3 & -2.155 \\
\hline & Pimeras diferencias & Constante & 2 & $-5.119 *_{-}$ \\
\hline
\end{tabular}

Nota: *Estadísticamente significativo al $1.00 \%$

Fuente: Elaboración propia 
hipótesis nula es la raíz unitaria según el test de ADF (Augmented Dickey-Fuller). Se encontró que todas las series son estacionarias en primeras diferencias.

Como siguiente paso, con el objetivo de evaluar si existe heterocedasticidad condicional autorregresiva, se llevó a cabo la prueba del Multiplicador de Lagrange de Engle. De acuerdo con este test, se rechaza la hipótesis nula de que no hay efectos ARCH (1). Por lo tanto, no se puede rechazar

CUADRO 1

PRUEBA SOBRE RAÍZ UNITARIA (TEST DE ADF)

\begin{tabular}{|c|c|c|c|c|}
\hline Variable & Tipo & $\begin{array}{c}\text { Componentes } \\
\text { prueba }\end{array}$ & Rezagos & $\mathrm{ADF}$ \\
\hline \multirow[t]{3}{*}{ Productividad total de los factores } & Niveles & Constante & 3 & -0.814 \\
\hline & Niveles & Constante $y$ tendencia & 3 & -1.68 \\
\hline & Pimeras diferencias & Constante & 2 & $-4.227 *_{-}$ \\
\hline \multirow[t]{3}{*}{ Inflación } & Niveles & Constante & 3 & -1.178 \\
\hline & Niveles & Constante $y$ tendencia & 3 & -0.541 \\
\hline & Pimeras diferencias & Constante & 2 & $-4.029 *_{-}$ \\
\hline \multirow[t]{3}{*}{ Apertura Comercial(X+M/PB) } & Niveles & Constante & 3 & -1.819 \\
\hline & Niveles & Constante $y$ tendencia & 3 & -1.099 \\
\hline & Pimeras diferencias & Constante & 2 & $-6.358-$ \\
\hline \multirow[t]{3}{*}{ IED (Inversión externa directa / PIB) } & Niveles & Constante & 3 & -0.97 \\
\hline & Niveles & Constante $y$ tendencia & 3 & -2.246 \\
\hline & Pimeras diferencias & Constante & 2 & $-6.869-$ \\
\hline \multirow[t]{3}{*}{ Profundidad Financiera (M2 / PIB) } & Niveles & Constante & 3 & -0.289 \\
\hline & Niveles & Constante $y$ tendencia & 3 & -2.171 \\
\hline & Pimeras diferencias & Constante & 2 & $-4.210^{*}$ \\
\hline \multirow[t]{3}{*}{ Profundidad Financiera (M1 / PIB) } & Niveles & Constante & 3 & -0.846 \\
\hline & Niveles & Constante $y$ tendencia & 3 & -2.155 \\
\hline & Pimeras diferencias & Constante & 2 & $-5.119 *$ \\
\hline
\end{tabular}

Nota: *Estadísticamente significativo al $1.00 \%$

Fuente: Elaboración propia

la presencia del efecto ARCH sobre el crecimiento de la PTF, incluso en el 6\% de nivel; esto es, periodos de alta (baja) volatilidad de la PTF están seguidos por periodos de alta (baja) volatilidad. Los resultados pueden observarse en el cuadro 2 .

Como siguiente paso, con el objetivo de evaluar si existe heterocedasticidad condicional autorregresiva, se llevó a cabo la prueba del Multiplicador de Lagrange de Engle. De acuerdo con este test, se rechaza la hipótesis nula de que no hay efectos ARCH (1). Por lo tanto, no se puede rechazar 


CUADRO 2
PRUEBA DEL MULTIPLICADOR DE LAGRANGE DE ENGLE
\begin{tabular}{lrrr}
\hline Rezagos & $\mathrm{x}^{2}$ & Grados de libertad & Prob $>\mathrm{x}^{2}$ \\
\hline 1 & 6.799 & 1 & 0.0091 \\
2 & 7.743 & 2 & 0.0208 \\
3 & 7.647 & 3 & 0.0539 \\
4 & 10.948 & 4 & 0.0272 \\
\hline Fuente: Elaboración propia & &
\end{tabular}

la presencia del efecto ARCH sobre el crecimiento de la PTF, incluso en el 6\% de nivel; esto es, periodos de alta (baja) volatilidad de la PTF están seguidos por periodos de alta (baja) volatilidad. Los resultados pueden observarse en el cuadro 2.

Asimismo, para evaluar si es el modelo más apropiado en un VECM o un VAR, se llevó a cabo las pruebas de cointegración de Johansen. La hipótesis nula es la existencia de cointegración de grado $(0,1,2,3$ y 4$)$, las cuales son consistentes en señalar que se rechaza esta hipótesis. Por tanto, como las variables no están cointegradas, no se puede correr un modelo VECM y lo correcto es utilizar un modelo VAR.

Como no existe cointegración, no existe causalidad de largo plazo; sin embargo, si existe causalidad de corto plazo de las variables independientes a la variable dependiente. Las pruebas de Wald sobre causalidad de Granger indican que existe causalidad de corto plazo de la inflación y la apertura comercial sobre la PTF. No obstante, cuando se mide el grado de apertura de la economía como la proporción de la IED como porcentaje del PIB y la profundidad financiera como M1 dividido entre el PIB, existe causalidad en el sentido de Granger de corto plazo de la inflación sobre la PTF.

De seguido, se llevó a cabo la prueba de Jarque-Bera con el objetivo de determinar si los residuos están o no normalmente distribuidos. Los resultados indican que se rechaza la hipótesis nula de que los residuos no están distribuidos normalmente para las variables de apertura comercial e inflación. Para el caso de la PTF y la profundidad financiera, los resultados muestran que no se rechaza la hipótesis nula descrita.

Los resultados del modelo ARCH-GARCH se presentan en el cuadro 3. La variable dependiente es el crecimiento de la PTF y los resultados muestran que:

a. Tasas de inflación más elevadas señalan un crecimiento menor de la PTF.

CUADRO 3

MODELO ARCH-GARCH (VARIABLE DEPENDIENTE: CRECIMIENTO DE LA PTF)

\begin{tabular}{lrr}
\hline \multicolumn{3}{c}{ Ecuación en medias } \\
\hline Inflación & -0.092 & $2.98^{*}$ \\
Volatilidad apertura comercial & 0.3744 & $4.48^{*}$ \\
Volatilidad profundidad financiera & -0.2637 & $1.77^{* *}$ \\
Constante & 0.0247 & $5.56^{*}$ \\
\hline \multicolumn{1}{c}{ Varianzas } \\
\hline ARCH & -0.0673 & 0.16 \\
GARCH & -0.3908 & 0.02 \\
Constante & -0.0014 & 0.75 \\
\hline $\mathrm{N}$ & 57 & \\
Distribución & t-student \\
Wald $x^{2}(3)$ & 25.1 & \\
Prob $>x^{2}$ & 0.0000 & \\
\hline
\end{tabular}

Fuente: Elaboración propia 
b. Un mayor crecimiento de los flujos de comercio (las exportaciones más las importaciones) dentro del PIB incrementa la PTF.

c. Una mayor volatilidad de la profundización financiera disminuye la PTF.

Por último, para establecer si el modelo utilizado está especificado de manera correcta, se procedió a realizar las siguientes pruebas econométricas utilizando los residuos correspondientes. En primer lugar, los valores p de un correlograma con 26 rezagos están por encima del $5 \%$, por lo que se puede rechazar la hipótesis nula de que no existe correlación serial de los residuos. En segundo lugar, utilizando la prueba Portmanteau $(Q)$ para ruido blanco (igual a 32,32 y Prob> $\chi 2$ $(26)=0.1827)$ se rechaza la hipótesis nula citada anteriormente, por lo que los residuos son ruido blanco. Finalmente, el estadístico Bartlett sobre periodograma acumulativo indica que se rechaza la hipótesis nula nuevamente, por lo que los residuos son ruido blanco. Estos resultados se pueden observan en el gráfico 7.

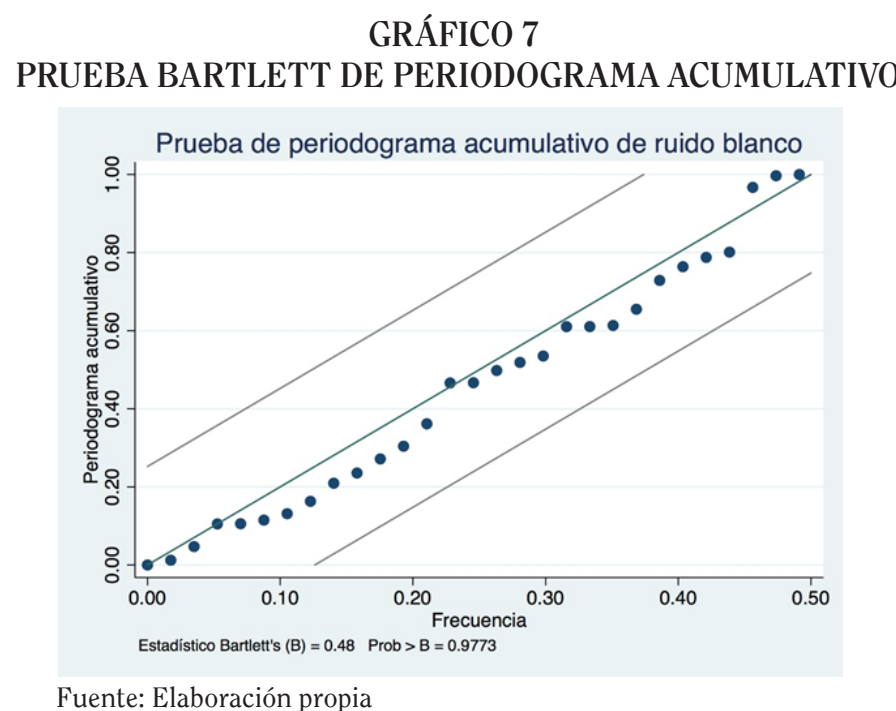

\section{.IV. CONCLUSIONES}

Al igual que lo muestra la amplia evidencia empírica para otros países, en Costa Rica existe una asociación positiva entre el crecimiento de la productividad total de los factores y el crecimiento económico. De hecho, en el presente estudio, durante el periodo de 1960 a 2019, el $69 \%$ de la variación en el crecimiento económico es explicado por cambios en el crecimiento de la productividad total de los factores.

El resto del cambio del crecimiento económico se debe a las variaciones en la contribución de los factores productivos. Tanto la acumulación de capital como el mayor uso del factor trabajo han contribuido al crecimiento económico en todas las décadas sin excepción. No obstante, el aporte del factor trabajo al crecimiento económico ha sido por la cantidad y no por la calidad de la mano de obra. Solamente entre 2010 y 2019, la mayor calidad del trabajo empleado ha aportado positivamente al crecimiento económico. Este periodo coincide con un estancamiento en el número de empleos que crea la economía y una disminución en la tasa de participación de la población económicamente activa, lo cual es indicativo de que salen de la fuerza de trabajo, en términos relativos, personas menos capacitadas.

Durante los años sesenta y setenta, la productividad en Costa Rica experimentó un crecimiento importante, al igual que lo hizo la producción. No obstante, este crecimiento estuvo asociado al modelo de sustitución de importaciones y tuvo el obstáculo que se basó en la acumulación 
de factores, en lugar de la innovación y la reducción de costos reales. Como consecuencia, la productividad se estancó al final de este periodo e hizo inviable mantener ese modelo de crecimiento económico.

En los últimos 40 años, a partir de 1977, el crecimiento de la productividad en Costa Rica ha sido más la excepción que la regla. Al dividir el periodo en décadas, el país tuvo crecimientos de la productividad solo en los noventa y los dos mil diez. Es más, la crisis económica que sufrió el país a inicios de los ochenta, causó un retroceso en el nivel de productividad, el cual tardó 30 años en recuperarse, pues el país recobró el mismo nivel de productividad que tenía en 1977 hasta el año 2007.

El cambio de modelo de crecimiento económico, primero al de promoción de exportaciones y luego al de atracción de inversión extranjera directa, ha sido positivo en términos de generación de crecimiento de la productividad, la cual muestra una clara tendencia positiva. Esta tendencia solo se ha visto interrumpida por las crisis internacionales de inicios de la década de los 2000 y de 2008.

La inestabilidad macroeconómica afecta de forma negativa el crecimiento de la productividad en Costa Rica. Este trabajo muestra evidencia de que la productividad del país se desmejora con niveles altos de inflación. Por su parte, la productividad se ve afectada positivamente por un mayor crecimiento de la apertura comercial y negativamente por una mayor volatilidad de la profundización financiera.

\section{AGRADECIMIENTOS}

Se agradece la colaboración de Gilberto Arce Alpizar en la estimación del modelo econométrico. Las interpretaciones y conclusiones son responsabilidad del autor.

\section{REFERENCIAS}

Abarca G., A. \& Ramírez V., S. (2016). Estudio del Crecimiento Económico Costarricense, 1960-2014. Observatorio del Desarrollo, Universidad de Costa Rica. https://odd.ucr.ac.cr/ estudio-del-crecimiento-economico-costarricense-1960-2014/\#: :text=Estudio\%20del\%20 Crecimiento\%20Econ\%C3\%B3mico\%20Costarricense\%2C\%201960\%2D2014,-E1\%20 presente $\% 20$ trabajo\&text $=\mathrm{Se} \% 20$ encuentra $\% 20$ que $\% 201 \mathrm{a} \% 20$ naturaleza,cuantas $\% 20$ similares $\% 20$ el $\% 20$ crecimiento\%20econ $\%$ C3\%B3mico

Acemoglu, D., \& Zilibotti, F. (1997). Was Prometheus unbound by chance? Risk, diversification, and growth. Journal of Political Economy, 105(4), 709-51. https://doi.org/10.1086/262091

Ackerberg, D. A., Caves, K. \& Frazer, G. (2015). Identification Properties of Recent Production Function Estimators. Econometrica, 83(6), 2411-2451. https://doi.org/10.3982/ECTA13408

Alfaro, L., Kalemli-Ozcan, S., \& Sayek, S. (2009). FDI, Productivity and Financial Development. World Economy, 32(1), 111-35. https://doi.org/10.1111/j.1467-9701.2009.01159.x

Angeletos, G. M. (2007). Uninsured idiosyncratic investment risk and aggregate saving. Review of Economic Dynamics, 10(1), 1-30. https://doi.org/10.1016/j.red.2006.11.001

Aschauer, D. A. (1989). Is Public Expenditure Productive? Journal of Monetary Economics, 23(2), 177-200. https://doi.org/10.1016/0304-3932(89)90047-0.

Barro, R. J. (2001). Human Capital and Growth. The American Economic Review, 91(2), 12-17. https://doi.org/10.1257/aer.91.2.12 
Bauer, P. W. (1990). Decomposing TFP growth in the presence of cost inefficiency, nonconstant returns to scale, and technological progress. Journal of Productivity Analysis, 1(4), 287-299. https://doi.org/10.1007/BF00160047

Berument, H., Nergiz Dincer, N. N., \& Mustafaoglu, Z. (2011). Total factor productivity and macroeconomic instability. The Journal of International Trade \& Economic Development, 20(5), 605-629. https://doi.org/10.1080/09638190903365930

Beveren, I. V. (2010). Total Factor Productivity Estimation: A Practical Review. Journal of Economic Surveys, 26(1), 98-128. https://doi.org/10.1111/j.1467-6419.2010.00631.x

Bronzini, R., \& Piselli, P. (2009). Determinants of long-run regional productivity with geographical spillovers: The role of R\&D, human capital and public infrastructure. Regional Science and Urban Economics, 39(2), 187-199. https://doi.org/10.1016/j.regsciurbeco.2008.07.002

Chang, R., Kaltani, L., \& Loayza, N. V. (2009). Openness can be good for growth: The role of policy complementarities. Journal of Development Economics, 90(1), 33-49. https://doi. org/10.1016/j.jdeveco.2008.06.011

Coe, D. T., Helpman, E., \& Hoffmaister, A. W. (1997). North-South R \& D Spillovers. The Economic Journal, 107(440), 134-149. https://doi.org/10.1111/1468-0297.00146

Cukierman, A., \& Meltzer, A. H. (1986). A theory of ambiguity, credibility, and inflation under discretion and asymmetric information. Econometrica, 17, 1099-1128. https://doi. org/10.2307/1912324

Dotsey, M., \& Sarte, P. D. (2000). Inflation uncertainty and growth in a cash-in-advance economy. Journal of Monetary Economics, 45(3), 631-655. https://doi.org/10.1016/s03043932(00)00005-2

Easterly, W. \& Levine, R. (2001). What have we learned from a decade of empirical research on growth? It's Not Factor Accumulation: Stylized Facts and Growth Models. The World Bank Economic Review, 15(2), 177-219. https://doi.org/10.1093/wber/15.2.177

Erosa, A., Koreshkova, T., \& Restuccia, D. (2010). How Important Is Human Capital? A Quantitative Theory Assessment of World Income Inequality. The Review of Economic Studies 77(4), 14211449. https://doi.org/10.1111/j.1467-937X.2010.00610.x

Evers, M., Niemann, S., \& Schiffbauer, M. (2008). Inflation, financial market incompleteness and long-run TFP-growth. Working Paper, Bonn Graduate School of Economics.

Friedman, M. (1977). Nobel Lecture: Inflation and Unemployment. Journal of Political Economy, 85(3), 451-472. https://doi.org/10.1086/260579

Froyen, R. T., \& Waud, R. N. (1987). An Examination of Aggregate Price Uncertainty in Four Countries and Some Implications for Real Output. International Economic Review, 28(2), 353-372. https://doi.org/10.2307/2526730

Hafer, R. W. (1986). The Response of Stock Prices to Changes in the Weekly Money and the Discount Rate. Review, 68(3), 5-14. https://doi.org/10.20955/r.68.5-14.hzb

Hahn, F. H. (1970). Savings and uncertainty. The Review of Economic Studies 37(1), 21-24. https:// doi.org/10.2307/2296495

Harberger, A. C. (1990). Reflections on the Growth Process [Working paper]. University of California.

Harberger, A. C. (1993). The Process of Economic Growth: A Vision for the 1990s. [Paper presentation] Conference of the Argentine Bankers' Association (ADEBA). University of California, Los Angeles.

Harberger, A. C. (1998). A Vision of the Growth Process. The American Economic Review, 88(1), 1-32. https://www.jstor.org/stable/116816 
Holland, A. S. (1986). Wage Indexation and the Effect of Inflation Uncertainty on Employment: An Empirical Analysis. The American Economic Review, 76(1), 235-243. https://www.jstor.org/ stable/1804141

Ivankovich, G. \& Martínez Castillo, J. (2020). La Productividad en Costa Rica. Estudios sobre productividad. (Serie programa visión para el desarrollo, no. 24). Academia de Centroamérica. https://www.academiaca.or.cr/programa-vision/la-productividad-en-costa-rica/

Jorgenson, D. W. (1995a). Productivity Volume 1: Postwar U.S. Economic Growth. The MIT Press.

Jorgenson, D. W. (1995b). Productivity Volume 2: International Comparisons of Economic Growth. The MIT Press.

Jorgenson, D. W., Gollop, F. M., \& Fraumeni, B. M. (1987). Productivity and U.S. Economic Growth. Harvard University Press. https://doi.org/10.1016/c2009-0-11949-9

Juster, F. T., \& Taylor, L. D. (1975). Towards a theory of saving behavior. American Economic Review, 65(2), 203-209. https://www.jstor.org/stable/1818853

Juster, F. T., \& Wachtel, P. (1972a). Inflation and the consumer. Brookings Papers on Economic Activity, 1972(1), 71-121. https://doi.org/10.2307/2534115

Juster, F. T., \& Wachtel, P. (1972b). A note on inflation and the saving rate. Brookings Papers on Economic Activity, 1972(3), 765-778. https://doi.org/10.2307/2534131

Kendrick, J. W. (1956). Productivity Trends: Capital and Labor. The Review of Economics and Statistics, 38(3), 248-257. https://doi.org/10.2307/1925777

Kendrick, J. W. (1961). Productivity Trend in the United States. Princeton University Press.

Kendrick, J. W., \& Grossman, E. S. (1980). Productivity in the United States: Trends and Cycles. The Johns Hopkins University Press.

Kim, Y. E., \& Loayza, N.V. (2017). Productivity and its Determinants: Innovation, Education, Efficiency, Infrastructure, and Institutions. [Working paper]. DECRG Kuala Lumpur Seminar Series. https://www.worldbank.org/en/events/2017/11/28/productivity-and-itsdeterminants-innovation-education-efficiency-infrastructure-and-institutions

Kim, Y. E., \& Loayza, N. V. (2019). Productivity Growth Patterns and Determinants across the World. (Policy Research Working Papers, 8852). World Bank. https://doi.org/10.1596/18139450-8852

Levine, R., Loayza, N., \& Beck, T. (2000). Financial intermediation and growth: Causality and causes. Journal of Monetary Economics, 46(1), 31-77. https://doi.org/10.1016/s03043932(00)00017-9

Lucas, R. (1973). Some International Evidence on Output-Inflation Tradeoffs. The American Economic Review, 63(3), 326-34. https://www.jstor.org/stable/1914364

Maiti, D. (2013). Market imperfections, trade reform and total factor productivity growth: Theory and Practices from India. Journal of Productivity Analysis 40(2), 207-218. https://doi. org/10.1007/s11123-012-0313-z

Monge, R. (2019). Productividad y crecimiento económico: Experiencias de algunos países de reciente desarrollo. (Serie Programa Visión para el Desarrollo, PV-03-19), Academia de Centroamérica.

Montalbano, P., Federici, A., Triulzi, U., \& Pietrobelli, C. (2005). Trade openness and vulnerability in Central and Eastern Europe. (WIDER working papers, 2005/43). United Nations UniversityWorld Institute for Development.

Park, D., Abiad, A., Estrada, G., Han, X., \& Tian, S. (2017). Transcending the middle-income challenge. En Asian Development Outlook (ADO) 2017: Transcending the Middle-Income Challenge (pp. 47-109). Asian Development Bank. https://doi.org/10.22617/FLS178632-3 
Plastina, A., \& Lence, S. H. (2018). A Parametric Estimation of Total Factor Productivity and Its Components in U.S. Agriculture. American Journal of Agricultural Economics, 100(4), 10911119. https://doi.org/10.1093/ajae/aay010

Robles Cordero, E. (2000). Economic Growth in Central America: Evolution of Productivity in Manufacturing. (HIID Development Discussion Papers, No. 749). Cambridge, Harvard University.

Rodríguez-Clare, A.; Sáenz, M.; y Trejos, A. (2003). Análisis del crecimiento económico de Costa Rica. (Serie de Estudios Económicos y Sectoriales, RE2-03-001). Banco Interamericano de Desarrollo.

Rodrik, D. (1998). Why do more open economies have bigger governments? Journal of Political Economy 106(5), 997-1032. https://doi.org/10.1086/250038

Scarpetta, S., \& Tressel, T. (2002). Productivity and convergence in a panel of OECD industries: Do regulations and institutions matter? (OECD Economics Department Working Papers, no. 342). Organisation for Economic Co-operation and Development. https://doi. org/10.1787/18151973

Shackleton, R. (2013). Total Factor Productivity Growth in Historical Perspective. (Working Paper series, 2013-01) Congressional Budget Office, Washington, D.C. https://www.cbo.gov/ publication $/ 44002$

Sickles, R. \& Zelenyuk, V. (2019). Measurement of Productivity and Efficiency: Theory and Practice. Cambridge University Press. https://doi.org/10.1017/9781139565981

Solow, R. M. (1957). Technical Change and the Aggregate Production Function. The Review of Economics and Statistics, 39(3), 312-320. https//doi.org/10.2307/1926047

Stigler, G. J. (1947). Trends in Output and Employment. National Bureau of Economic Research. https://www.nber.org/books-and-chapters/trends-output-and-employment

Straub, S. (2008). Infrastructure and Growth in Developing Countries : Recent Advances and Research Challenges. (Policy Research Working Papers, No 4460). The World Bank. https// doi.org/10.1596/1813-9450-4460

Tinbergen, J. (1942). Zur Theorie der Langfristigen Wirtschaftsentwicklung. Weltwirtschaftliches Archiv, 55(1), 511-549. https://www.jstor.org/stable/40430851

Tsionas, M. G. \& Polemis, M. L. (2019). On the estimation of total factor productivity: A novel Bayesian non-parametric approach. European Journal of Operational Research, 277(3), 886902. https://doi.org/10.1016/j.ejor.2019.03.035

Young, A. (1992). A Tale of Two Cities: Factor Accumulation and Technical Change in Hong Kong and Singapore. NBER Macroeconomics Annual, 7, 13-64. https://doi.org/10.1086/654183

Zhang, J., Jiang, C., \& Wang, P. (2014). Total Factor Productivity and China's Miraculous Growth: An Empirical Analysis. (SSRN Scholarly Papers, ID 2456009). Social Science Research Network. https://doi.org/10.2139/ssrn.2456009

Este artículo se encuentra disponible mediante la licencia Creative Commons Reconocimiento-NoComercial-SinObraDerivada 3.0 Costa Rica. Para mayor información escribir a revista.iice@ucr.ac.cr. 